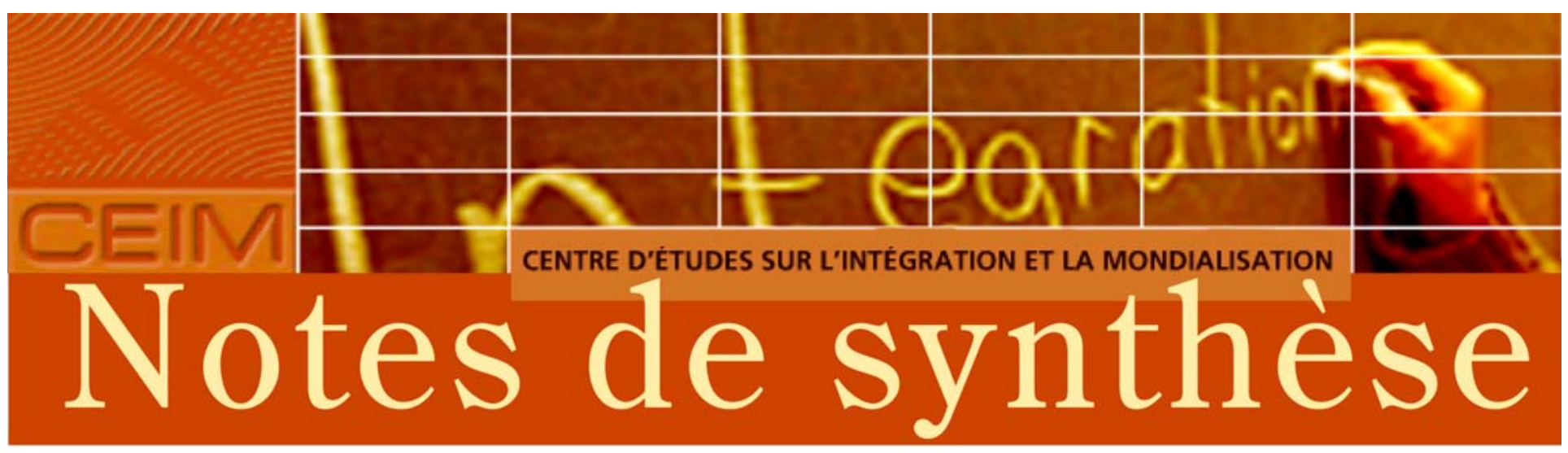

Juillet 2009

\title{
La muleta du protectionnisme
}

\author{
Christian Deblock
}

Le commerce résiste mal à la crise. Les prévisionnistes de l'OMC, de l'OCDE et du FMI ne s'entendent pas sur les chiffres, mais tous s'accordent à dire que le commerce mondial connaîtra en 2009 sa pire année depuis la Guerre. Le scénario catastrophe se profile à l'horizon : la baisse de la production et de l'emploi ne peut qu'inciter les pays à protéger leur économie contre la concurrence étrangère et à faire preuve d'une agressivité accrue pour trouver des débouchés. Résister à la tentation du protectionnisme et ne pas chercher à exporter la crise chez les autres, mais, au contraire, soutenir le commerce et aider les plus pauvres à passer à travers la crise, c'est le mot d'ordre que sont donnés les grands de ce monde, appuyés en cela à l'unisson par les dirigeants des grandes organisations internationales, l'OMC en tête. Discours politique de circonstance certes, mais qui résiste mal aux faits et nous fait oublier que le commerce, comme la finance, n'obéit qu'à une seule loi, le profit. De leur côté, si les économistes insistent pour dire que le protectionnisme est aussi contre-productif que néfaste, on ne les entend guère, par contre, quand il est question de commercialisme. Et pour cause ! Le discours tourne depuis trois décennies autour d'une formule magique, l'intégration compétitive dans l'économie mondiale. La compétitivité n'est seulement devenue qu'une dangereuse obsession, comme s'en inquiétait déjà Paul R. Krugman dans les années 1990 ; elle a surtout peu à voir avec l'image du "doux commerce" chère à Montesquieu. C'est sur ces deux facettes du débat que je me propose de revenir dans cette note. Je reviendrai dans un premier temps sur le discours " officiel " et sur les arguments qui lui sont sous-jacents, puis dans un deuxième temps sur le ton pondéré que les économistes cherchent à maintenir dans un débat qui ne porte déjà plus sur le protectionnisme, mais sur le commercialisme. Je conclurai sur cette idée. 


\section{L'ombre du protectionnisme...}

Depuis le début de la crise, chaque grande réunion internationale est devenue l'occasion pour les dirigeants des grands pays de la planète de lancer un message fort: il n'est pas question de refaire les mêmes erreurs que par le passé en cédant au protectionnisme et au nationalisme économique. Retour sur quelques déclarations ${ }^{1}$.

- Déclaration finale du sommet du G20 sur les marchés financiers et l'économie mondiale tenue à Washington le 15 novembre 2008 : les chefs d'État et de gouvernement s'engagent à s'abstenir dans les douze mois à venir "d'ériger de nouvelles barrières à l'investissement ou au commerce des biens et des services, d'imposer de nouvelles restrictions ou de mettre en œuvre des mesures de stimulation des exportations contraires aux règles de l'OMC ». Promesse est également faite de tout mettre en œuvre pour relancer et conclure le cycle de Doha et s'engager à agir de manière responsable pour soutenir le système d'échanges mondiaux. Ils convenaient enfin que les mesures de régulation financière qui seraient mises en place n'étoufferaient pas l'innovation et encourageraient le développement des échanges de produits et de services financiers.

- $\quad$ Sommet du G20 de Londres des 2 et 3 avril 2009: les chefs d'État et de gouvernement s'engagent, à nouveau, "à prendre toutes les mesures nécessaires" pour " promouvoir le commerce et l'investissement mondiaux et rejeter le protectionnisme, dans le but de soutenir la prospérité ». Cet engagement se fait toutefois plus précis que dans la déclaration précédente. Prenant acte de la baisse brutale du commerce mondial et «des pressions protectionnistes grandissantes", les dirigeants jugent " essentiel de redynamiser le commerce et l'investissement à l'échelle internationale " et de ne pas commettre «l'erreur de retomber dans le protectionnisme comme par le passé ».

À l'engagement réaffirmé de ne pas dresser de nouveaux obstacles à l'investissement et au commerce, et ce, jusqu'à la fin de 2010, viennent s'y ajouter deux nouveaux : (1) le déblocage d'un fonds de 250 milliards de dollars pour appuyer le financement du commerce et l'appui réitéré aux pays en développement pour les aider à relancer le commerce ; et (2) la réduction au minimum des effets sur le commerce et l'investissement des plans d'action nationaux (ce qui comprend les politiques budgétaires et les aides au secteur financier), l'OMC et les organes internationaux ayant le mandat de surveiller le respect de cet engagement et de publier des rapports trimestriels à cet égard. Les dirigeants du G20 ont par ailleurs réaffirmé leur détermination "à parvenir à un résultat ambitieux et équilibré dans le Cycle de Doha pour le développement $»^{2}$.

- Autre déclaration: celle des ministres des Finances et des gouverneurs des banques centrales du G8 à l'Aquila (Italie) le 13 juin 2009. La déclaration note avec satisfaction que "jusqu'ici, malgré la crise économique, les grands pays ont globalement résisté aux pressions politiques grandissantes qui s'exercent en faveur de réactions protectionnistes" et que "les gouvernements honorent les engagements qu'ils ont pris de résister au protectionnisme en matière d'investissement ». 
- Dernière déclaration à cette date, celle du G8 à l'Aquila le 9 juillet 2009. Les dirigeants y réaffirment solennellement leur double engagement de résister à " la tentation protectionniste » et de faire " la promotion de la libéralisation des marchés pour le commerce et l'investissement». Ils soulignent également "l'importance de respecter l'engagement de maintenir le statu quo renouvelé à Londres, lequel vise à ne pas prendre de mesures qui dresseraient des obstacles au commerce et à l'investissement et à remédier promptement à la situation dans le cas contraire". Une attention particulière est accordée aux mesures qui viendraient entraver la libre circulation des capitaux, notamment vers les pays en développement. La déclaration reconnaît d'ailleurs le rôle de l'investissement comme "source de croissance, d'emploi, d'innovation et de développement " tout en faisant référence au développement durable et à "la responsabilité sociale des entreprises ». Enfin, les dirigeants expriment leur détermination à " mener à bien» le cycle de Doha et à conclure les négociations en 2010 .

\section{Les organisations internationales sur la même longueur d'onde}

Renoncer à

l'ouverture des marchés n'est pas

la solution à la

crise économique

Pascal Lamy, Washington, 24 avril 2009
Le même discours est tenu par les dirigeants des grandes organisations internationales, solidaires comme jamais en ces temps de crise. Le plus actif est, comme il se doit, le directeur général de l'OMC, Pascal Lamy : il est omniprésent dans tous les forums internationaux, tire sans relâche la sonnette d'alarme, et a su habilement tourner la crise actuelle à l'avantage de l'organisation qu'il dirige ${ }^{3}$. Dans quelle mesure son message sera-t-il écouté et les négociations de Doha relancées ? L'avenir le dira, mais reconnaissons-lui au moins le mérite de vouloir redorer le blason de l'OMC et du commerce.

L'OMC n'est pas la seule organisation à se faire alarmiste et à se porter à la défense d'un système commercial ouvert. Ainsi, le 5 mars 2009, le Secrétaire général de l'OCDE, Angel Gurria, invitait les pays à ne pas céder à la facilité et tomber dans la politique du chacun-pour-soi, mais plutôt d'ouvrir les marchés et de saisir l'occasion pour réactiver les réformes commerciales ${ }^{4}$. Même son de cloche du côté de la Banque mondiale dont le président Robert $\mathrm{B}$. Zoellick appelle les dirigeants à ne pas "céder aux chants des sirènes des solutions protectionnistes, que ce soit pour le commerce, les plans de relance ou ceux de sauvetage" et à se souvenir que "l'isolationnisme économique peut conduire à un enchaînement négatif d'évènements comme on l'a vu dans les années $30 \ldots »^{5}$. Ou encore du côté de la CNUCED, dont le secrétaire général Supachai Panitchpakdi qualifie le nationalisme économique "d'intention louable ", mais dangereuse. Et celui-ci de s'inquiéter de l'affaiblissement de la confiance dans le système commercial, mais aussi des effets très négatifs de la chute du commerce et de l'investissement sur les économies des pays en développement ${ }^{6}$.

Ces multiples déclarations étaient manifestement destinées à sensibiliser les dirigeants politiques et l'opinion publique avant le sommet du G20 de Londres, mais leur degré de convergence n'en est pas moins étonnant : il y a péril dans la demeure. 


\section{Les médias agitent l'épouvantail, les économistes se mobilisent}

Les organisations internationales ne sont pas les seules à s'inquiéter de la résurgence du protectionnisme en ces temps de crise. Les médias se font aussi l'écho de ces préoccupations, les uns pour évoquer le spectre de crise de $1929^{7}$, d'autres le retour du nationalisme économique ${ }^{8}$, et d'autres encore le respect des engagements multilatéraux ${ }^{9}$.

Les économistes se mobilisent également, comme ils l'avaient fait en 1930 contre la loi Smoot-Hawley, mais très rares sont, malgré tout, ceux qui sont prêts à comparer la situation actuelle à celle des années trente. Par exemple, pour le grand historien libéral Douglas A. Irwin ${ }^{10}$, non seulement le monde d'aujourd'hui est-il fort différent de celui des années trente, mais les dirigeants politiques semblent bien déterminés à ne pas répéter les erreurs du passé. Il faut demeurer vigilant, mais, constate-til, les gouvernements ont acquis de l'expérience en matière de gestion des crises et disposent aujourd'hui d'instruments qu'ils n'avaient pas à l'époque. Les tarifs douaniers d'aujourd'hui ne se comparent pas non plus à ceux de cette époque, et ce, même si les gouvernements disposent toujours d'une certaine latitude en la matière ${ }^{11}$. De plus, et contrairement aux années trente, les gouvernements doivent composer avec les traités internationaux et les règles de l'OMC. Aussi imparfaites que soient ces règles, elles ont pour elles le mérite d'exister, d'être contraignantes et d'obliger les gouvernements à prendre en considération les risques qu'ils prennent en les violant. Une autre réalité, c'est que l'économie mondiale a profondément changé : il faut désormais composer avec les multinationales et leurs réseaux d'activité qui traversent les frontières. Enfin, et contrairement aux années trente, les pays ont appris à coopérer et les grandes puissances, les États-Unis en tête, entendent conserver une attitude responsable, conscientes qu'elles sont de l'exemple qu'elles montrent aux autres.

En somme, nous avons deux types de discours qui se croisent: l'un se veut alarmiste, l'autre rassurant. Comment faire la part des choses ? Comment, surtout, y voir clair ? Essayons de démêler les choses, mais auparavant revenons sur ces deux discours et essayons d'en dégager les idées maîtresses. Commençons par le discours des "politiques".

\section{Cinq sources d'inquiétude}

Pour aller à l'essentiel, on peut dégager cinq points de convergence ou sources d'inquiétude dans le discours politique.

(1) Il ne s'agit pas de se tromper de cible : l'économie est entrée dans une ère de turbulence financière et de repli général de l'activité économique, mais la responsabilité n'en incombe pas «à une défaillance des marchés et du libre-échange ». Le commerce n'est en rien responsable de la situation actuelle. Tout au contraire, aussi bien la réussite économique des pays émergents que l'augmentation du niveau de vie dans de nombreux pays montrent que le commerce est non seulement un agitateur de développement, mais également un puissant instrument d'intégration à l'économie mondiale ${ }^{12}$. Aux dires de Pascal Lamy, " L'expansion continue du commerce est une garantie contre l'instabilité du marché et les turbulences financières». Et celui-ci d'ajouter qu'«alors que l'expansion du commerce constitue une partie visible de la mondialisation, la crise de l'économie financière ne démontre que trop clairement que la 
mondialisation devrait s'accompagner d'un solide ensemble de règles internationales convenues et applicables, protégeant tous les pays contre les mouvements cycliques. Ainsi l'OMC fournit des éléments nécessaires de gouvernance mondiale en vue de maîtriser les conséquences négatives de la mondialisation ${ }^{13}$. En clair, non seulement le commerce est un puissant garant de stabilité dans une économie mondiale en turbulence, mais l'OMC, avec ses disciplines fortes et son expérience acquise en matière de coopération multilatérale, devrait être une source d'inspiration pour la refonte du système financier international.

(2) Le protectionnisme pointe à l'horizon. Pour le moment encore on ne peut parler que de soubresauts, mais les périodes de recul de l'activité économique sont toujours propices à sa recrudescence, et, si la situation économique devait s'aggraver davantage, on peut craindre que les gouvernements ne soient tentés de céder aux pressions politiques. Certains signes ne trompent pas.

Peu avant le sommet du G-20 de Londres ${ }^{14}$, la Banque mondiale publiait fort opportunément une brève étude faisant état de l'accroissement des mesures à caractère protectionniste prises par les gouvernements entre novembre 2008 et février $2009^{15}$. La période est courte, mais symbolique puisque le 15 novembre 2008, les dirigeants du G-20 avaient affirmé leur engagement en faveur d'une économie mondiale ouverte et rejeté le protectionnisme. L'étude de la Banque mondiale constate qu'en un peu plus de quatre mois, les mesures de restriction au commerce se sont rapidement multipliées. Ses auteurs relèvent que pas moins de 17 pays appartenant au G-20 ont mis en place pas moins de 47 mesures dont l'effet serait de restreindre le commerce. Les pays développés en ont mis en place 12, et les pays en développement 35 , les premiers préférant soutenir les exportations et les seconds limiter les importations. L'étude ne prend pas en compte les mesures antidumping et les mesures compensatoires qui ont, elles aussi, connu de fortes poussées. Les auteurs restent prudents, l'économie mondiale d'aujourd'hui, avec ses chaînes de valeur, ses interdépendances et ses règles juridiques, ayant peu à voir avec celle des années trente, mais cette poussée de fièvre n'en est pas moins préoccupante.

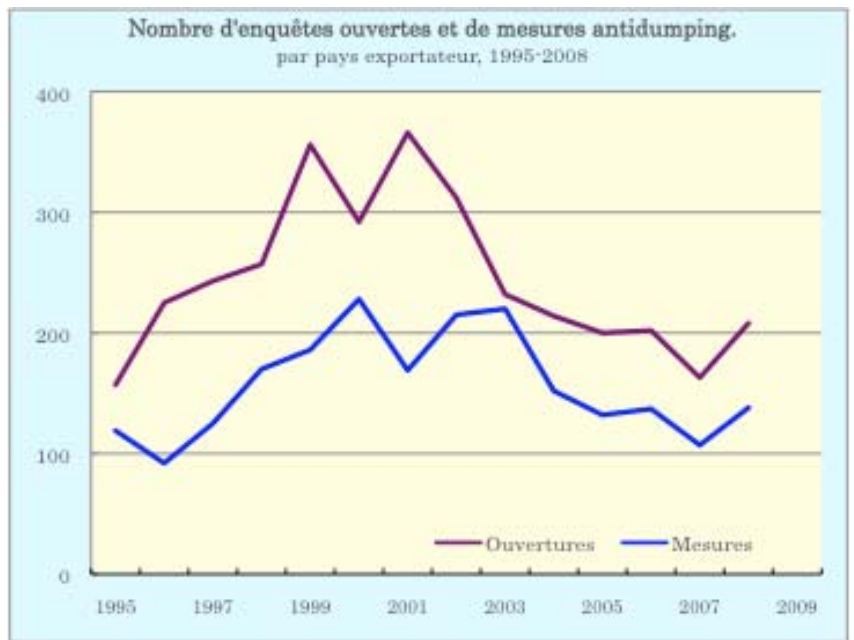

Ce constat est partagé par l'OMC, qui relève de son côté une nette augmentation du nombre de demandes d'enquêtes et de poursuites antidumping 16 : il y aurait eu 208 ouvertures d'enquête en 2008, dont 120 entre juillet et décembre 2008 (103 en 2007 pour la même période). L'Inde est le pays qui en a ouvert le plus grand nombre (42 pour les six derniers mois) ; viennent ensuite le Brésil, la Chine et la Turquie. La Chine est le pays le plus visé ; elle est suivie par la Communauté européenne, les États-Unis, Taiwan et la Thaïlande. 
(3) Il faut soutenir le commerce et, surtout, tuer dans l'œuf la spirale de la dépression dans laquelle il paraît engagé. Les données ont, effectivement, de quoi inquiéter: non seulement le volume du commerce mondial a chuté brutalement, mais il semble surréagir au recul de l'activité économique dans les pays développés, avec des impacts particulièrement violents sur les économies les plus ouvertes, notamment celles des pays émergents et en développement. Ainsi, les experts de l'OMC parlent d'une baisse des exportations mondiales de l'ordre de $9 \%$ en 2009 (un recul de $10 \%$ pour les pays développés et de 2 à $3 \%$ pour les pays en développement).

Pays émergents choisis, taux de variation du PIB réel

Amérique latine et Asie, 2004-2009
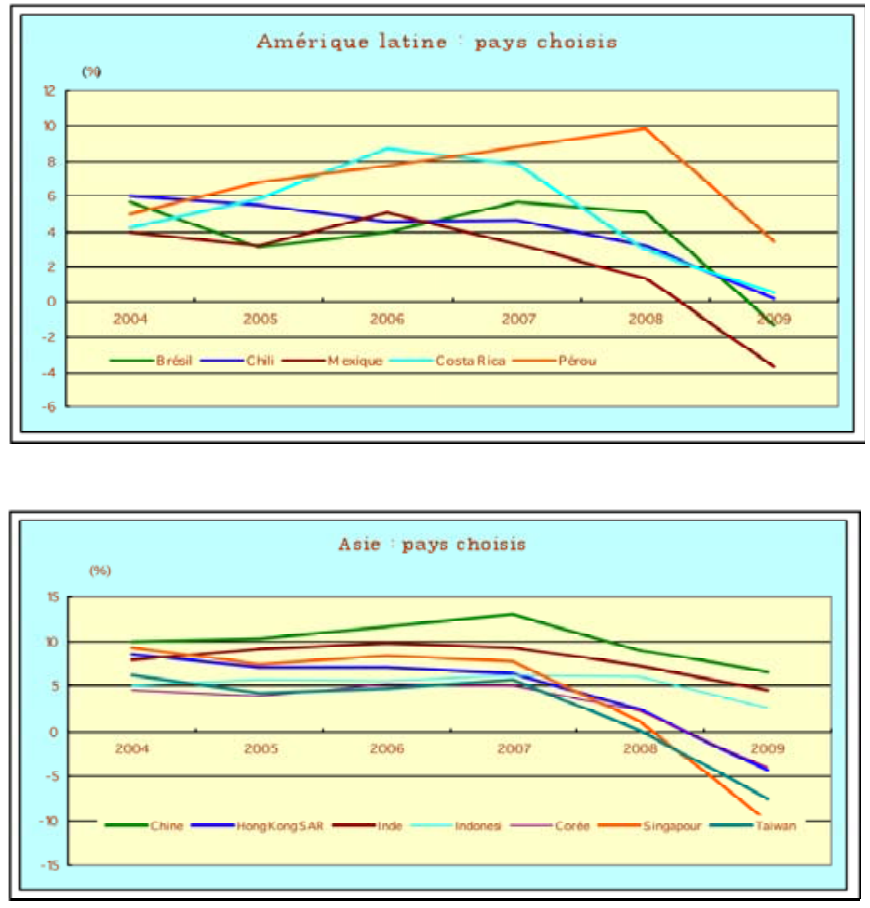

Sources : FMI, World Economic Database, avril 2009
L'OCDE se fait plus pessimiste encore, l'organisme anticipant une chute du volume des échanges internationaux en 2009 de l'ordre de $13 \%$ par rapport à 2008. Trois facteurs paraissent avoir un effet amplificateur: (1) la diminution des crédits à l'exportation dans la mouvance de la crise de liquidités, (2) la gestion des stocks en flux serrés et l'effet en amont de la baisse de la demande sur les chaînes de valeur ${ }^{17}$, et (3) l'effondrement des marchés de matières premières.

Le commerce n'est pas le seul secteur de l'économie mondiale qui est touché par la crise économique. Les flux financiers le sont également, en particulier les transferts d'argent des travailleurs migrants et les investissements internationaux. Ainsi, la CNUCED estime que la crise devrait entraîner une baisse de quelque $20 \%$ des flux d'investissements directs étrangers en 2008, et sans doute davantage encore en 2009. Et ce, même si les pays en développement semblent mieux résister $(3,6 \%)$ et que les flux en destination de l'Asie de l'Est et du SudEst (la moitié des flux à destination des pays en développement) continuent de progresser à un taux modeste. Ces prévisions sont corroborées par l'Institute of International Finance pour qui les flux de capitaux à destination des pays émergents devraient tomber à 165 milliards de $\$$ en 2009, comparativement à 466 millions de $\$$ en 2008 et un record de 929 milliards de $\$$ en 2007. Le dernier Rapport sur le financement $d u$ développement dans le monde (juin 2009) parle, quant à lui, d'un effondrement des flux de capitaux privés: ils chuteraient de 707 milliards de $\$$ en 2008 à 363 milliards de $\$$ en 2009 , des chiffres qui sont très loin de ceux qui avaient été atteints en 2007 , soit 1,2 billion de $\$$.

Partant de ces constats, des mesures d'urgence s'imposent, pour deux raisons principales. Tout d'abord, le commerce et les investissements soutiennent la croissance de l'économie mondiale et permettent à de nombreux pays en développement de tirer leur épingle du jeu d'une intégration plus étroite aux marchés mondiaux. Ensuite, l'expérience 
historique montre que les échanges internationaux et les mouvements de capitaux sont extrêmement sensibles aux variations de l'activité économique mondiale: locomotive de la croissance en période de prospérité, les échanges internationaux surréagissent en temps de crise. Les priorités vont à la régulation des marchés financiers, mais elles doivent aussi aller à la reprise du commerce, et celle-ci demande des mesures fiscales et monétaires de grande ampleur pour stimuler vigoureusement la demande intérieure et remettre l'activité économique sur les rails de la croissance. Les plans de relance américains ou chinois, par exemple, vont dans cette direction, mais attention toutefois : leur mise en œuvre ne doit pas se faire au détriment des engagements internationaux ni conduire à l'instauration de nouvelles barrières à l'investissement et au commerce des biens et des services.

(4) La préférence nationale. C'est la quatrième source d'inquiétude, la moins visible et la plus difficile à contrer. De nombreux signes montrent en effet qu'en cherchant à soutenir leurs propres industries et leur économie, les gouvernements ne sont pas insensibles à deux arguments politiques: la défense de l'emploi et l'utilisation des fonds publics. Si certains s'inquiètent de l'ampleur des déficits publics, de la croissance de la dette et des injections massives de liquidités, d'autres s'inquiètent de l'utilisation des argents publics, et d'autres encore des restructurations en cours sur l'emploi. Pour dire les choses simplement: l'opinion publique demande des comptes aux gouvernements et, surtout, des résultats. Dans un tel contexte, la tentation est grande, pour les gouvernements, de jouer la préférence nationale, notamment lorsqu'il s'agit de contrats publics et de subventions. Et elle est d'autant plus grande que les disciplines commerciales ne sont pas aussi fortes ni aussi étendues dans ces deux domaines qu'elles ne le sont pour les tarifs douaniers par exemple.

Ainsi, les critiques et les menaces n'ont pas tardé à fuser après l'annonce par les États-Unis ${ }^{18}$ et la France de mesures de soutien à leurs industries nationales, mais tous les pays ne font pas nécessairement preuve de la même transparence. La Chine est également particulièrement pointée du doigt, l'opacité des procédures d'appel d'offre pour les programmes de relance disqualifiant pratiquement les entreprises étrangères ${ }^{19}$. Mais le mouvement s'étend : en Asie, au détriment des entreprises chinoises d'ailleurs, et en Amérique latine. Des pressions se font également sentir pour imposer certaines exigences aux investissements étrangers ou, à l'inverse, pour inciter les entreprises nationales à investir et à produire sur place plutôt qu'à l'étranger.

(5) Le commerce n'est pas responsable des inégalités. À l'exception notable de l'OIT, plus réservé, un consensus existe parmi les organisations internationales, à savoir que le commerce n'est pas responsable de l'accroissement des inégalités; s'il faut chercher des coupables, il vaut mieux regarder du côté des changements technologiques, mais aussi, un langage nouveau, du côté de la mondialisation financière et de la déréglementation des flux de capitaux à l'échelle internationale ${ }^{20}$.

Il faut, en effet, apporter une distinction dans la mondialisation entre ces deux univers fort différents que sont celui du commerce d'un côté et celui de la finance de l'autre. Ainsi, selon le FMI, et contrairement 
à la croyance populaire, le commerce aurait permis de réduire les inégalités tant dans les pays développés que dans les pays en développement. Il n'en va pas de même avec la mondialisation financière : les flux et les mouvements de capitaux ont augmenté les inégalités. Par contre, alors que le FMI n'y voit qu'un phénomène transitoire qui pourrait être corrigé par des politiques d'éducation, des politiques actives d'emploi et des financements adéquats, l'Institut international d'études sociales de l'OIT adopte dans son rapport de 2008 une attitude beaucoup plus tranchée ${ }^{21}$.

Pour l'organisme, la déréglementation internationale des flux de capitaux n'a permis d'améliorer ni la productivité ni la croissance de l'emploi ; elle a surtout engendré beaucoup d'instabilité et de précarité dans les conditions de travail, renforçant ainsi la tendance à la baisse de la part des salaires en affaiblissant le pouvoir de négociation des travailleurs et en mettant beaucoup de pression sur les systèmes de protection sociale. L'OIT évite cependant de remettre en question le consensus sur le commerce, et ce, même si certaines interrogations avaient surgi à ce sujet dans le rapport de 2004 de la Commission mondiale sur les dimensions sociales de la mondialisation $^{22}$. Par contre, il y a un point sur lequel tout le monde s'accorde : la crise va aggraver la pauvreté et les inégalités dans les pays en développement. L'OIT en annonce la couleur: la crise pourrait jeter dans le chômage quelque 30 millions de personnes dans le monde (voire 50 millions selon un scénario plus pessimiste), dont 23 millions dans les pays en développement. Quant au nombre de personnes dans la misère, il pourrait augmenter de 50 à 90 millions du seul fait de la crise ${ }^{23}$.

Crise alimentaire, crise énergétique, crise financière, crise commerciale ; les pays en développement ont été durement touchés en peu de temps. Ces crises successives n'ont pas seulement pour effet de briser l'élan du développement des dernières années et d'augmenter la pauvreté et le sous-emploi structurels ; elles remettent directement en question la réalisation des Objectifs de développement pour le millénaire (ODM). Les retards pris avant la crise étaient déjà inquiétants, mais selon le dernier Rapport de suivi mondial (2009) de la Banque mondiale, ils sont maintenant d'autant plus alarmants que les effets de la crise risquent d'être de longue durée, notamment pour tout ce qui touche au développement humain (scolarité, santé, etc.). Au point que des mesures d'urgence s'imposent pour venir en aide aux pays les plus pauvres et les plus vulnérables. Le G20 a fait un pas dans cette direction en demandant aux Nations Unies de mettre en place un mécanisme de surveillance et de soutien particulier pour ces pays. L'OIT a procédé de même en leur accordant une attention particulière dans le Pacte mondial pour l'emploi que la Conférence générale a adopté à la mi-juin. Il n'en demeure pas moins que pour les auteurs du rapport de suivi des ODM, «Le commerce est un puissant moteur de la croissance et de la réduction de la pauvreté dans les pays en développement, et donc un facteur de progrès vers la réalisation des ODM. C'est pourquoi le maintien et l'élargissement de l'accès de ces pays aux marchés internationaux doivent être au cœur des objectifs de développement ». 


\section{Prendre la mesure des choses}

Une fois n'est pas coutume, les économistes, bien que préoccupés par la tournure des évènements, préfèrent tenir un discours plus rassurant comme je l'ai déjà indiqué. Ils ne sont certainement pas mécontents de voir, d'un côté, les organisations internationales alerter l'opinion publique et inviter les gouvernements à ne pas céder à la facilité, et de l'autre, les dirigeants politiques des grandes puissances économiques, les anciennes comme les nouvelles, réaffirmer leurs engagements et afficher une belle unanimité en faveur d'une économie mondiale ouverte. Par contre, les faits et l'expérience du passé les inclinent à tempérer l'alarmisme du discours politique et à adopter une attitude plus mesurée. Les arguments d'Irwin reflètent assez bien cette attitude générale, même si, bien sûr, ils ne sont nécessairement pas partagés par tous. On peut les ramener à trois principaux.

(1) Le protectionnisme est obsolète. Il est, à cet égard, assez significatif que le seul argument sérieux, la recrudescence des poursuites antidumping, ne soit guère très robuste. Qu'on en juge simplement ! Les périodes de récession et de recul de l'activité économique ont toujours été accompagnées d'un recours accru à l'antidumping, mais dans le cas présent, on ne peut guère parler d'explosion du phénomène. Le nombre de nouvelles enquêtes a été, certes, supérieur à 2007 (163), mais les chiffres sont assez comparables à ceux de 2006 (202) et très en deçà de ceux observés en 2001 (366), année de forte récession. Elles proviennent aussi davantage des "nouveaux utilisateurs ", notamment les pays émergents, que des utilisateurs traditionnels. Il y a trois causes à cela.

- Tout d'abord, l'un des résultats les plus remarquables du système GATT/OMC est d'avoir considérablement réduit les tarifs douaniers et d'avoir ainsi ouvert les marchés. Aussi bien que, les tarifs douaniers moyens sont passés de $16 \%$ à $3,7 \%$ entre 1952 et 2005 dans le cas des États-Unis, de $17 \%$ à $4,2 \%$ dans le cas du RoyaumeUni et de $11 \%$ à $3,8 \%$ dans le cas du Canada. Il est difficile d'avoir des chiffres précis sur les tarifs moyens avant la signature du GATT, mais l'OMC estime que lorsque le GATT fut signé en 1947, les tarifs moyens des parties contractantes variaient entre 20 et $30 \%$. La diminution des tarifs est tout aussi marquée pour les pays émergents, d'autant qu'elle a le plus souvent été unilatérale.

Droits de douane moyens

Produits non agricoles

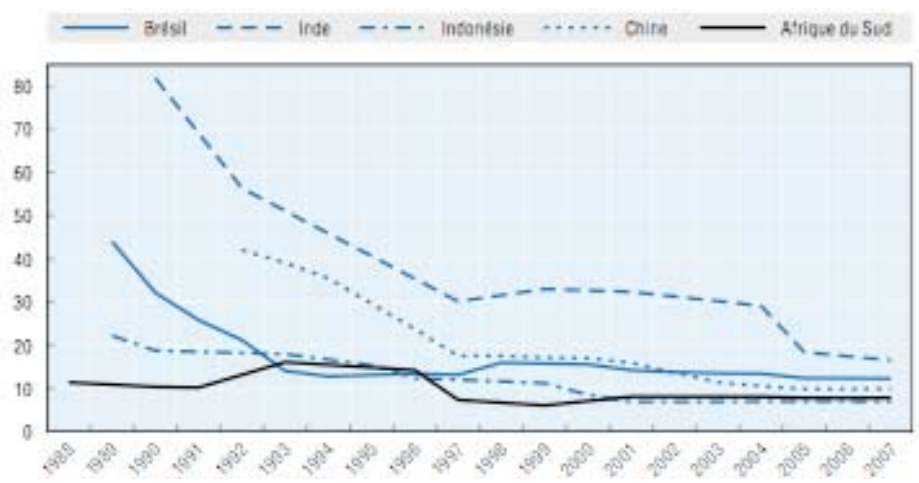

OCDE, "La mondialisation et les économies émergentes ", Synthèses, mars 2009.
Toujours est-il que la baisse générale des tarifs n'a pas été sans conséquence sur le degré d'ouverture des économies concernées. En prenant un indicateur aussi simple de cette ouverture que la part des exportations dans le PIB mondial, on constate qu'elle est passée de $5,5 \%$ à $19,4 \%$ entre 1950 et $2005^{24}$. Que signifie cette augmentation ? Sinon, d'une part, que les exportations ont augmenté quatre fois plus vite que le PIB, et d'autre part, que les économies de la planète ne sont plus seulement interdépendantes, mais engagées dans un processus durable d'intégration. 
- Ensuite, les utilisateurs traditionnels ont pris conscience non seulement de la difficulté d'engager des procédures antidumping ou de sauvegarde, mais également des coûts que cela peut entraîner lorsque le commerce est croisé, intra-branche et que les intrants des produits exportés proviennent de l'étranger ${ }^{25}$. Comment dans ce cas protéger certaines industries sans pénaliser les industries d'exportation ou le secteur de la distribution importateur?

- Enfin, concernant les nouveaux utilisateurs, un constat s'impose: les marchés du Sud sont beaucoup plus sensibles à la concurrence internationale que ceux du Nord, surtout lorsqu'elle vient d'autres pays du Sud, met en péril les industries d'exportation et menace les gains du commerce réalisés grâce aux spécialisations dans les secteurs à forte intensité de main-d'œuvre. Ajoutons que face au recul de la demande dans les pays du Nord, la tentation est grande de détourner les flux du commerce et d'écouler les surplus de production vers les marchés voisins ou les plus vulnérables. Les nombreux différends commerciaux qui ont

\begin{tabular}{|llll|}
\multicolumn{4}{c}{ Nombre d'enquêtes ouvertes } \\
1995-2008 \\
Pays initiateur & \multicolumn{2}{c|}{ Pays visé } \\
\hline Inde & 564 & Chine & 677 \\
Etats-Unis & 418 & Corée & 252 \\
Com. Europ. & 391 & Etats-Unis & 189 \\
Argentine & 241 & Taiwan & 187 \\
Afrique du Sud & 206 & Indonésie & 145 \\
Australie & 197 & Japon & 144 \\
Brésil & 170 & Thaïlande & 142 \\
Chine & 151 & Inde & 137 \\
Canada & 145 & Russie & 109 \\
Turquie & 137 & Brésil & 97 \\
Source : OMC & & & \\
\hline
\end{tabular}
opposé le Brésil et l'Argentine et mis le MERCOSUR sous pression au début de la décennie, ou encore le nombre actuel de différends dont fait l'objet la Chine de la part de pays asiatiques, illustrent le phénomène. Celui-ci demeure malgré tout limité et constitue un argument de plus pour maintenir les marchés du Nord ouverts et conclure le cycle de Doha.

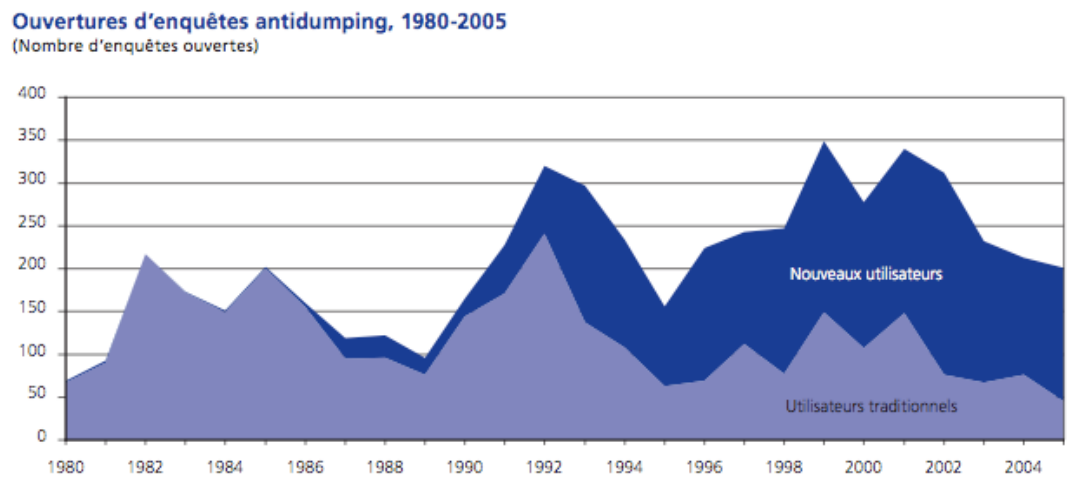

Note: Les utilisateurs traditionnels sont notamment l'Australie, le Canada, les CE et les Etats-Unis.

Source: Prusa (2005) et Secrétariat de l'OMC.

(2) Le système commercial possède ses pare-feux ${ }^{26}$. Les disciplines commerciales sont loin d'être parfaites, tout comme elles sont loin de couvrir les multiples dimensions de l'économie mondiale, mais elles ont le mérite d'exister, d'avoir considérablement progressé depuis la signature du GATT en 1947 et, surtout, d'être respectées, à la grande satisfaction des entreprises d'ailleurs. Trois constats peuvent être faits. 
- Tout d'abord, contrairement au GATT qui n'était dans le fond qu'un gentleman agreement, l'OMC est une véritable organisation internationale dotée d'un réel pouvoir sur ses membres. Le règlement des conflits commerciaux par la voie de l'arbitrage obligatoire est incontestablement l'un des grands acquis du cycle d'Uruguay. Le mécanisme est loin d'être parfait, mais tous les observateurs sont d'avis qu'il fonctionne relativement bien, qu'il contribue au renforcement de la règle de droit dans les relations commerciales entre les membres, qu'il accélère et facilite les procédures en cas de différend, et qu'il n'y a pas de biais en faveur de tel ou tel plaignant. Il est à cet égard significatif que, depuis la création de l'OMC, près des deux tiers des plaintes aient débouché sur la formation d'un groupe spécial, que près de $30 \%$ des cas aient abouti sur un règlement conforme aux décisions arbitrales et seulement $20 \%$ des décisions sur des menaces de rétorsion commerciale ${ }^{27}$.

- Ensuite, les disciplines commerciales s'étendent aux barrières non tarifaires ${ }^{28}$. Timidement amorcées pendant le cycle Kennedy, les négociations ont rapidement progressé pendant le cycle de Tokyo, pour s'étendre davantage encore pendant le cycle d'Uruguay. Les différents " codes" et accords conclus ont, ainsi, permis de rendre le commerce plus transparent, de réduire un tant soit peu le protectionnisme invisible et d'abaisser ainsi les coûts de transaction, les coûts d'entrée du marché. Les effets sont sans doute moins spectaculaires que les réductions tarifaires ; ils sont également difficilement quantifiables, mais on estime néanmoins que les barrières non tarifaires étaient dans les années 1990 trois fois moins importantes qu'à la fin des années 1970 et que leur impact sur l'organisation des activités transnationales est beaucoup plus significatif que la diminution des droits de douane ${ }^{29}$.

Les zones grises restent cependant nombreuses et les résistances très vives. Non seulement parce que les codes et accords dans ce domaine affectent directement le pouvoir de légiférer et de réglementer des États, mais parce que les règles ont été édictées par les pays développés, du moins largement inspirées par celles qu'ils se sont données pour niveler le terrain de la concurrence sur leurs propres marchés. L'accord sur les marchés publics illustre le problème.

Peu de pays en développement ou émergents ont signé l'accord et les négociations avec les grands pays émergents, dont la Chine et le Brésil, sont au point mort, un avantage dont ces pays tirent tout le parti possible à l'heure de la relance pour favoriser leurs propres entreprises, tout en dénonçant le protectionnisme des autres ${ }^{30}$. Mais, là encore, il faut prendre la mesure des choses: on ne peut en même temps accorder à ces pays un traitement spécial et différencié et dénoncer certaines pratiques qui, pour être fort discutables, ne remettent pas pour autant en question les engagements qui ont été pris au terme des négociations d'Uruguay ou au moment de leur adhésion à l'OMC. Le système commercial multilatéral est un système intégré, et non un système à la carte ; on peut donc présumer - avec une fausse naïveté - que les pays qui ne sont pas encore signataires des accords plurilatéraux finiront par se rallier à l'avant-garde...

- Enfin, les négociations commerciales d'Uruguay ont permis de faire progresser les discussions autour de nouveaux domaines, en particulier les services commerciaux, les investissements, la 
propriété intellectuelle ou la concurrence. La question du travail a été balayée par les ministres du Commerce lors de la Conférence de Singapour et renvoyée à l'OIT, et les questions de l'investissement, de la concurrence et de la transparence des marchés ne font plus partie du programme de Doha, la facilitation des échanges étant la seule des quatre "questions de Singapour" à continuer d'y figurer. Ces importantes concessions faites par les pays développés aux pays en développement n'ont toutefois pas empêché ces derniers de contourner le problème en prenant la voie des accords commerciaux régionaux - pour reprendre la terminologie de l'OMC -, et ce, même si la plupart d'entre eux sont bilatéraux.

Les effets négatifs de ces accords sont bien connus : fragmentation de l'économie mondiale, détournement d'attention vis-à-vis de l'OMC, exclusion, discrimination et détournement de commerce, enchevêtrement des règles, etc. On ne peut toutefois dénier leur contribution au développement du commerce ni les avantages qu'ils procurent aux participants. Certains ont même vu en eux un moyen de dénouer les impasses du multilatéralisme et de faire malgré tout progresser les règles commerciales. Jean-Marc Siroën a avancé récemment un nouvel argument en leur faveur : c'est un autre gardefou du système en temps de crise ${ }^{31}$. D'une part, ils limitent la marge de manœuvre des pays signataires et d'autre part, les liens commerciaux étroits qui unissent ces pays font tampon aux possibles mesures protectionnistes que ceux-ci seraient tentés de prendre. Siroën évoque le cas de l'Union européenne, dont $65 \%$ des importations proviennent des pays membres et $5 \%$ de pays avec lesquels elle a des accords commerciaux, mais on pourrait aussi prendre celui de l'ALENA : plus de $55 \%$ des exportations et plus du tiers des importations des trois pays (Canada, États-Unis et Mexique) se font à l'intérieur de la zone de libre-échange. Pour prendre le seul cas des États-Unis, ce n'est pas moins de $40 \%$ de leurs exportations de marchandises et le tiers de leurs importations qui sont couvertes par un accord de libre-échange ${ }^{32}$.

(3) La reprise économique pourrait venir plus vite qu'on ne le pense. L'ampleur de la crise financière et son impact foudroyant sur l'activité économique et le commerce mondial ont pris tous les prévisionnistes par surprise et les scénarii les plus pessimistes, pour ne pas dire fantaisistes, ont été avancés. Les chiffres du PIB et de l'emploi paraissent conforter une telle hypothèse, ce dont s'alarme d'ailleurs l'OIT, inquiète de voir remis en question son programme en faveur du travail décent. Depuis, les scénarii ont été réajustés et les prévisions économiques relevées. Michaël Mussa est un des premiers à avoir avancé l'hypothèse audacieuse d'une vigoureuse reprise dès l'automne 2009. Maintenant, c'est au tour de l'OCDE d'annoncer dans ses dernières prévisions économiques que "La phase de contraction de l'activité semble devoir bientôt toucher à sa fin dans la zone OCDE, après un recul sans précédent depuis la guerre. La reprise sera sans doute faible et fragile pendant un certain temps, et les conséquences économiques et sociales de la crise seront durables. Cela étant, les choses auraient pu être pires ${ }^{33}$. Trois facteurs justifieraient ce regain d'optimisme.

i. L'analyse systématique des dix récessions américaines depuis la Seconde Guerre mondiale permet de conclure que plus forte est la récession, plus courte elle est, et plus vigoureuse est la reprise économique ${ }^{34}$. 
ii. Ensuite, selon le FMI et l'OCDE, «le " découplage " ne semble avoir été qu'un mirage sur le chemin de la récession " (OCDE). À l'effondrement synchronisé de la production et des échanges devrait suivre une reprise synchronisée au vu des perspectives économiques meilleures que prévu de la Chine et de la plupart des grands pays émergents, mais aussi des États-Unis et, dans une moindre mesure, du Japon.

iii. Des plans de relance ambitieux et massifs ont été rapidement mis en place, autant pour soutenir le secteur financier et alimenter les circuits asséchés du crédit que pour soutenir la consommation et compenser par des

\begin{tabular}{|lcc|}
\hline \multicolumn{3}{|c|}{ PIB réel, pourcentage de variation } \\
\hline \multicolumn{1}{|c|}{$(\%)$} & 2009 & 2010 \\
États-Unis & $-2,8$ & 0,9 \\
Japon & $-6,8$ & 0,7 \\
Allemagne & $-6,1$ & 0,2 \\
Chine & 7,7 & 9,3 \\
Inde & 5,9 & 7,2 \\
Russie & $-6,8$ & 3,7 \\
Brésil & $-0,8$ & 4 \\
Source : $O C D E$, juin 2009 & & \\
\hline
\end{tabular}
investissements publics l'effondrement de l'investissement privé. Le plan de relance américain représente $5,6 \%$ du PIB et comprend, entre autres, des allègements fiscaux, des financements d'infrastructure, des subventions pour la recherche, l'éducation et la santé, des mesures d'aide sociale. Le plan chinois, annoncé en novembre 2008, représente quant à lui $10 \%$ du PIB. Il a également été opérationnalisé très rapidement. Il prévoit, entre autres, d'importants investissements en infrastructures et logements sociaux, des mesures de facilités bancaires et des mesures de soutien des revenus. Des mesures du même type ont été adoptées dans la plupart des grands pays émergents et au Japon. Seule l'Europe reste à la traîne, faute de coordination entre les plans de relance nationaux. Toujours est-il que les effets de ces plans commencent à se faire sentir, ce qui est somme toute encourageant.

Si le scénario d'une reprise rapide se confirme, cela mettrait un terme à la polémique sur le protectionnisme : une crise financière majeure suivie de la pire récession d'Après-Guerre, mais sans commune mesure néanmoins avec ce qui s'est passé dans les années Trente. Ce scénario modérément optimiste est plausible, mais fragile. Tout d'abord, le passé n'augure pas du futur. C'est l'une des leçons que l'on peut retenir des travaux de Wesley C. Mitchell sur les cycles économiques : chaque cycle "possède ses caractéristiques en propre". Tout comme chaque cycle s'accompagne de changements structurels, aussi petits soient-ils, qui font qu'une fois la reprise amorcée, l'économie ne revient jamais à l'état initial. Dans le cas présent, non seulement la crise est à bien des égards inédite, mais on peine à expliquer comment les mécanismes de la crise financière ont pu se transmettre aussi rapidement et brutalement aux circuits de l'économie réelle. $\mathrm{Y}$ a-t-il eu dérèglement contagieux, accumulation de déséquilibres partiels ou effet de structure, ou les trois en même temps?

L'attention a d'abord été portée sur les déséquilibres financiers, pour ne pas dire sur les malversations financières, puis dans un deuxième temps sur l'effondrement de la production, du commerce et des investissements. $\mathrm{Ne}$ faudrait-il pas aussi se demander pourquoi la contraction de l'activité 
économique, à l'image de la peste de la fable, ne touche pas tous les pays de la même façon et, surtout, pourquoi certaines économies résistent beaucoup mieux que d'autres ? Ainsi, si l'on prend les derniers chiffres publiés par la Banque mondiale pour la Chine, on constate que, malgré la chute des échanges commerciaux (une chute des exportations de $10 \%$ et des importations de près de $5 \%$ en 2009 par rapport à 2008 selon les projections), le solde de sa balance commerciale reste solidement positif. Par rapport à 2008, l'excédent est même en hausse : 62 milliards au premier trimestre 2009, contre 41 au premier trimestre de $2008^{35}$. De même, les réserves en devises n'ont augmenté que de 8 milliards de $\$$ au premier trimestre 2009 comparativement à 154 milliards en 2008 , mais elles augmentent malgré tout. On ne peut certainement pas en dire autant des États-Unis, qui continuent de s'endetter pour financer leur double déficit, et de la plupart des pays industrialisés, obligés qu'ils sont de s'endetter lourdement pour soutenir l'activité. Pour les seuls ÉtatsUnis, les besoins de financement sont estimés par l'OCDE à quelque $10 \%$ du PIB pour 2009 et à $11 \%$ pour 2010.

Enfin, même si l'Asie a été très touchée par la crise, on comprend mal pourquoi le FMI ou l'OCDE peuvent parler avec autant d'assurance de synchronisme des conjonctures. Une certaine prudence s'imposerait. La Chine et l'Inde, par exemple, affichent, malgré les problèmes, des taux de croissance fort enviables. Comme le faisait remarquer The Economist dans son édition du 21 mai 2009 ("Decoupling $2.0 »$ ), les économies émergentes, du moins les plus grosses, dépendent beaucoup moins des dépenses américaines qu'on ne le croit généralement. De plus, elles semblent dans l'ensemble répondre beaucoup mieux et avec beaucoup plus de détermination à la crise qu'on ne le pense. L'activisme de leurs gouvernements laisse aussi supposer qu'ils pourraient retrouver les chemins de la croissance plus rapidement et se démarquer ainsi plus radicalement encore du bloc occidental. Si tel est le cas, cela signifierait que ces pays, et surtout la Chine, verraient leur économie non seulement rebondir avant celles des pays industrialisés, mais sortir plus fortes encore de la crise. Mais qui va alors tirer qui ?

Taux de variation annuel du PIB américain, 1949-2009

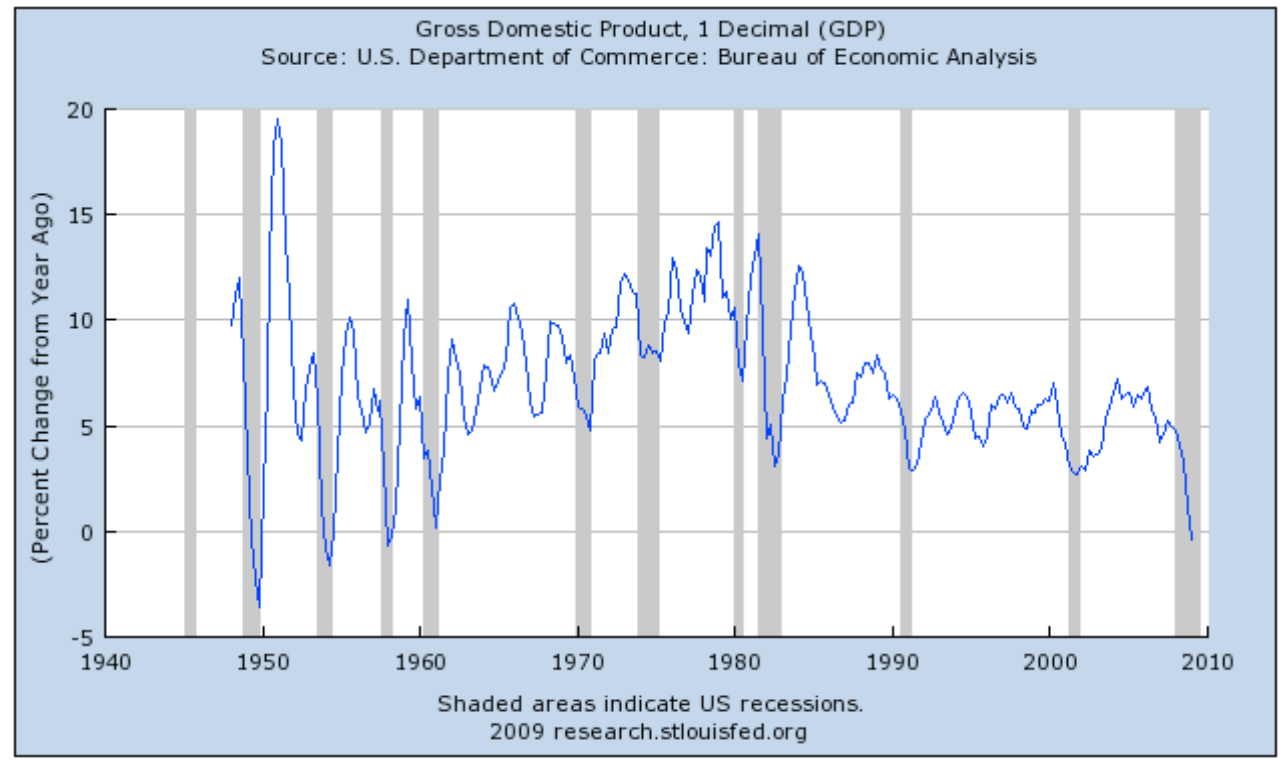

Source : Federal Reserve Bank of St-Louis 
En somme, il ressort des remarques précédentes que: (1) le protectionnisme - du moins traditionnel - tomberait en désuétude ; (2) malgré ses imperfections, le système commercial serait suffisamment robuste pour en contrôler les effets les plus nocifs ; et (3) malgré les incertitudes qui l'entourent, la reprise serait, en définitive, le meilleur antidote.

\section{Propos d'étape}

En définitive, si toute crise cyclique s'accompagne, dans une certaine mesure, d'un dérèglement des courants commerciaux de semblable nature, il est de moments comme en 1929 - où le déséquilibre structurel prend une importance toute nouvelle. Dans l'ordre des relations internationales, l'expression " crise de structure" peut être accueillie sans hésitation : le rééquilibre commanderait un profond réajustement des structures nationales ...Mais bientôt, toutes les données du problème apparaissent bouleversées. "Crise mondiale", "crise des échanges internationaux", "seconde révolution industrielle..." ", autant d'expressions soulignant que la réadaptation n'est plus possible dans le sens d'un retour à l'ancien système d'échanges internationaux".

(Jean Weiller, Problèmes d'économie internationale. Les échanges du capitalisme libéral. Paris, Presses universitaires de France, 1946, p. 192)
Étrange période que celle que nous traversons ! Alors que les cartes sont en train d'être redistribuées dans l'économie mondiale, les dirigeants politiques s'accrochent à l'OMC comme on s'accroche à une bouée de sauvetage tandis que de leur côté, les économistes préfèrent croire que l'alerte a été chaude, mais que, bientôt, tout devrait rentrer dans l'ordre et la crise n'être qu'un mauvais souvenir. Le débat actuel sur le protectionnisme est un mauvais débat. Non pas que le protectionnisme n'existe pas, ou que la tentation d'y recourir ne soit pas grande, mais parce que tout laisse croire qu'une page d'histoire est en train de se fermer et qu'au sortir de la crise, plus rien ne sera comme avant. Il y a malgré tout deux leçons que l'on peut tirer de ce débat.

Première leçon: la globalisation met à mal nos représentations traditionnelles de l'économie mondiale. Nous sommes habitués à voir dans le commerce un ensemble de flux reliant entre elles des nations souveraines. C'est la vision classique d'une économie inter-nationale avec ses relations d'interdépendance et sa division du travail fondée sur les avantages comparatifs. En introduisant les réseaux et les chaînes de valeur dans l'analyse, les économistes reconnaissent par là non seulement que l'intégration de l'économie mondiale passe moins par les États et leurs politiques d'ouverture que par les entreprises et l'organisation transnationale de leurs activités, mais aussi par le fait même que le protectionnisme n'est plus de notre temps. Il le fut peut-être à une époque où les relations commerciales gravitaient autour des États et où il était encore possible de penser l'économie sous l'angle national et de tracer une ligne de démarcation entre les deux espaces, l'espace national d'un côté et l'espace inter-national de l'autre. Aujourd'hui, ce n'est plus le cas, mais encore faut-il accepter ces nouvelles réalités et admettre que si la régulation du commerce est en train d'échapper aux États, d'autres approches que l'approche traditionnelle de la coopération intergouvernementale s'imposent.

Deuxième leçon: l'idée de "doux commerce" bute sur les intérêts commerciaux. Le libre commerce est tout sauf un bien commun. Que le commerce rapproche les sociétés et stimule les économies, personne ne le niera, mais cessons une fois pour toutes de penser que les déterminants du commerce seraient différents de ceux de la finance et que, contrairement à ce qui se passe sur les marchés financiers, le commerce et l'investissement seraient soumis à des règles. Des règles existent, mais, d'une part, elles ne s'appliquent pour l'essentiel qu'aux États et, d'autre part, elles ne visent qu'à favoriser la liberté du commerce et des investissements. La liberté du commerce est un postulat sur lequel ne plane pas l'ombre d'un doute dans les déclarations officielles et ce, même si derrière l'affirmation de cette liberté se profilent des intérêts bien sentis. Réaffirmer solennellement son engagement en faveur de la libéralisation des marchés n'est pas de la part des dirigeants politiques une preuve de sagesse. Il ne s'agit par là que de réaffirmer le statu quo, et ce, alors même que ce n'est pas de statu quo dont nous avons besoin, mais de règles, de règles sur la concurrence, sur la responsabilité des 
entreprises, sur les pratiques commerciales des entreprises, etc. Y auraitil deux globalisations: l'une déréglée et destructrice, celle de la finance, et l'autre, ordonnée et bénéfique, celle du commerce ? Poser la question, c'est déjà y répondre, mais encore faut-il se la poser ! 
Notes

${ }^{1}$ On retrouvera le texte des différentes déclarations sur le site du MAECI à la rubrique G8.

${ }^{2}$ Il y a néanmoins peu de chances que les choses bougent de ce côté là avant la fin de l'année ; au mieux peut-on s'attendre à une reprise des discussions sur les modalités des négociations. Voir à ce sujet le débat entre Jeffrey J. Schott et Arvind Subramanian en ligne sur le site du Peterson Institute for International Economics, « How to Revive the Global Trade Negotiations : A Debate », 25 avril 2009.

${ }^{3}$ Voir par exemple "Garder un commerce ouvert : résister à l'isolationnisme ", conférence du Directeur général de l’OMC, Pascal Lamy, Séoul, 23 février 2009.

${ }^{4}$ "Des marchés ouverts en temps de crise", allocution de M. Angel Gurria, Secrétaire général de l'OCDE, devant la Chambre de commerce internationale, Genève, 5 mars 2009. Le conseil exécutif de l'OCDE avait également joint sa voix, en novembre 2008, à celle de ceux qui voyaient poindre la menace protectionniste. L'OCDE a publié récemment un document d'information sur le commerce, Le commerce international : libre, équitable et ouvert?

${ }^{5}$ Communiqué de presse $n^{\circ}$ 2009/257/EXT, 17 mars 2009

6 " Le développement à la croisée des chemins : les perspectives des pays en développement dans un monde en crise ", 6 mars 2009.

${ }^{7}$ The Economist a été l'un des premiers à appuyer sur le bouton d'alarme en publiant dans son édition du 18 décembre 2008 un article sur la loi Smoot-Hawley (" The Battle of SmootHawley "). Il faut noter que, contrairement à la plupart de ceux qui évoquent l'épisode de la loi Smoot-Hawley, l'auteur de l'article prend la peine de retracer l'historique de cette loi et fait preuve de beaucoup de prudence quant aux conséquences qu'elle a eues tant sur le commerce international que sur les politiques de rétorsion.

8 Par exemple, Thomas Wieder, dans un article publié dans l'édition du Monde du 11 avril 2009, "Protectionnisme : la tentation permanente ", tournait en ridicule Colbert, Louis XIV et les marottes vestimentaires de l'époque, pour ainsi mieux souligner le caractère révolutionnaire du libre-échange comme principe de politique et, bien entendu, rappeler l'asphyxie du commerce international que provoqua le recours aux solutions "naturelles " (hausse des tarifs, dévaluation compétitive, etc.) dans les années trente. Voir également l'éditorial de The Economist en date du 7 février 2009, "The Return of Economic Nationalism ".

9 Par exemple l'article de Gary Clyde Hufbauer, «US Free Trade promises Must Be Honored", Financial Times, 29 mai 2009.

10 Douglas A. Irwin, "Trade Policy in 2008: Great Depression redux? », dans Richard Baldwin et Simon Evenett (dir.), What World Leaders Must Do to Halt the Spread of Protectionnism, Centre for Economic Policy Research, 2008, pp. 59-62 (en ligne).

11 Pascal Lamy a évoqué à plusieurs reprises le scénario catastrophe d'un alignement des tarifs douaniers sur les plafonds autorisés, autrement dit sur les tarifs consolidés. Si un tel scénario se produisait, il en résulterait une chute du commerce mondial de l'ordre de 7,7\%. (OCDE, « Keeping Markets Open at Times of Economic Crisis », Policy Brief, avril 2009).

12 OCDE, « La mondialisation et les économies émergentes », Synthèses, mars 2009.

13 Pascal Lamy, "L'expansion du commerce est une garantie contre les turbulences financières ", Geneva Lectures on Global Economic Governance, 6 février 2008.

${ }_{14}$ L'OMC a procédé de la même façon peu avant le sommet du G8 à L'Aquila en faisant circuler le rapport annuel sur l'examen des politiques commerciales, question d'enfoncer un peu plus le clou sur la progression du protectionnisme.

15 Elisa Gamberoni et Richard Newfarmer, "Trade Protection: Incipient but Worrisome Trends ", Banque mondiale, 4 mars 2009.

16 ETO Staff, "Report to the TPRB from the Director-General on the Financial and Economic Crisis and Trade Related Developments ", OMC, 23 janvier 2009.

${ }^{17}$ Voir par exemple pour le cas canadien la note de Erik Ens, "Le protectionnisme dans une économie mondialisée: les contrecoups de la politique du chacun pour soi» (Affaires étrangères et Commerce international Canada, 24 mars 2009).

18 "Le Buy American Act: une mauvaise idée ?», Chronique commerciale américaine, CEIM,

${ }_{19}$ Arnaud de la Grange, "Les étrangers écartés du plan de relance chinois ", Le Figaro, 28 mai 2009 , p. 21.

${ }^{20}$ FMI, World Economic Outlook : Globalization and Inequality, New York, 2007

21 International Institute for Labor Studies, World of Work Report 2008. Income Inequalities in the Age of Financial Globalization, Genève, 2008.

${ }^{22}$ Commission mondiale sur la dimension sociale de la mondialisation, Une mondialisation juste : créer des opportunités pour tous, Genève, BIT, 2004.

${ }^{23} \mathrm{La}$ Banque mondiale estime à 55 millions le nombre de personnes supplémentaires qui vivront avec moins de 1,25 \$ par jour (1 \$ en dollars PPA de 2005).

${ }^{24}$ En comparaison, cette part est passée de $4,6 \%$ en 1870 à $7,9 \%$ en 1913 . Source : OMC,

Rapport sur le commerce mondial 2007, Genève, 2007, pp. 263-264. 
${ }^{25}$ L'image traditionnelle selon laquelle l'économie serait divisée en deux parties, l'une qui serait composée d'industries orientées vers le marché intérieur et sensibles à la concurrence internationale et l'autre, d'industries hautement compétitives orientées vers les marchés internationaux, ne tient plus guère la route. La production est de plus en plus verticalement intégrée et les produits circulent de plus en plus d'un pays à l'autre. L'OCDE rappelle certains faits stylisés à propos des pays de l'OCDE : en moyenne aujourd'hui, le tiers des produits manufacturiers vendus sont des produits importés ; la part des intrants importés dans la production intérieure de biens est passée de $38 \%$ à $44 \%$ entre 1995 et 2005 ; les entreprises étrangères comptent, en moyenne, pour près du quart des emplois dans le secteur manufacturier.

26 Sur les pare-feux, voir Raymond J. Ahearn, The Global Economic Downturn and Protectionnism, Congressional Research Service, Washington, 23 mars 2009, R40461.

27 OMC, Rapport sur le commerce mondial 2007, op. cit. pp. 281 et suivantes.

${ }^{28}$ Les obstacles ou barrières non tarifaires comprennent les restrictions quantitatives, les subventions, les droits antidumping et les droits compensateurs, l'évaluation en douane ainsi que les normes et les règlements techniques.

${ }^{29}$ OMC, Rapport sur le commerce mondial 2008, Genève, 2008, pp. 88 et suivantes.

${ }^{30}$ Récemment, Pékin a ordonné, dans une directive, d'attribuer un maximum de contrats publics aux entreprises chinoises et d'exclure les entreprises étrangères des appels d'offres. La Chine est en bon droit de le faire puisqu'elle n'a jamais signé l'Accord de l'OMC sur les marchés publics (outre les 27 États membres de la Communauté européenne, seuls 12 pays sont parties à l'accord. La Chine est observatrice).

31 Jean-Marc Siroën, "Un retour du protectionnisme est-il à craindre ?», 25 mars 2009, en ligne.

${ }^{32}$ Christian Deblock, "Le bilatéralisme commercial des États-Unis ", à paraître, en ligne sur le site du CEIM, Montréal, 2008.

${ }^{33}$ OCDE, Perspectives économiques $n^{\circ} 85,24$ juin 2009.

34 Michaël Mussa, World Recession and Recovery: $A$ V or an L ?, Peterson Institute for International Economics, avril 2009.

${ }_{35}$ World Bank Office, Beijing, Quaterly Update, juin 2009. 